\title{
Shared genetic underpinnings of childhood obesity and adult cardiometabolic diseases
}

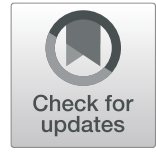

Fasil Tekola-Ayele ${ }^{1 *}$, Anthony Lee ${ }^{1}$, Tsegaselassie Workalemahu ${ }^{1}$ and Katy Sánchez-Pozos ${ }^{2}$

\begin{abstract}
Background: Obesity during childhood can lead to increased risk of adverse cardiometabolic diseases such as type 2 diabetes and coronary artery disease during adult life. Evidence for strong genetic correlations between child and adult body mass index (BMI) suggest the possibility of shared genetic effects. We performed a test for pleiotropy (shared genetics) and functional enrichment of single nucleotide polymorphisms (SNPs) associated with childhood $\mathrm{BMI}$ and 15 adult cardiometabolic traits using a unified statistical approach that integrates pleiotropy and functional annotation data.

Results: Pleiotropic genetic effects were significantly abundant in 13 out of 15 childhood BMl-adult cardiometabolic trait tests $\left(P<3.3 \times 10^{-3}\right)$. SNPs associated with both childhood BMI and adult traits were more likely to be functionally deleterious than SNPs associated with neither trait. Genetic variants associated with increased childhood obesity tend to increase risk of cardiometabolic diseases in adulthood. We replicated 39 genetic loci that are known to be associated with childhood BMI and adult traits (coronary artery disease, HDL cholesterol, myocardial infarction, triglycerides, total cholesterol, type 2 diabetes, BMI, waist circumference, and waist-to-hip ratio) in previous genomewide association studies. We also found a novel association of rs12446632 near GPRC5B, which is highly expressed in adipose tissue and the central nervous system, with adult HDL cholesterol.

Conclusions: This study found significant pleiotropic genetic effects and enrichment of functional annotations in genetic variants that were jointly associated with childhood obesity and adult cardiometabolic diseases. The findings provide new avenues to disentangle the genetic basis of life course associations between childhood obesity and adult cardiometabolic diseases.

Keywords: Genetic pleiotropy, Functional loci, Childhood obesity, Cardiometabolic diseases, Developmental Origins of Health and Disease (DoHAD)
\end{abstract}

\section{Background}

Obesity in childhood is increasingly becoming a significant global public health burden [1]. Several studies have documented that higher childhood body mass index (BMI), an established measure of obesity, is associated with increased risk of adverse cardiometabolic outcomes in adulthood such as type 2 diabetes, hypertension, dyslipidemia, coronary artery disease, and markers of cardiovascular diseases [2-13]. Identifying common biological pathways underlying childhood adiposity and

\footnotetext{
* Correspondence: ayeleft@mail.nih.gov

${ }^{1}$ Epidemiology Branch, Division of Intramural Population Health Research, Eunice Kennedy Shriver National Institute of Child Health and Human Development, National Institutes of Health, 6710B Rockledge Drive, Room 3204, Bethesda, MD 20892-7004, USA

Full list of author information is available at the end of the article
}

adult diseases will help unravel mechanisms linking childhood BMI and adult cardiometabolic diseases. It will also provide insights that will help distinguish adiposity-related biological processes that operate in childhood from those that operate in adulthood and to formulate possible causal relationships.

Recent evidence for strong genetic correlations between childhood BMI and a few adulthood cardiometabolic traits such as BMI $[14,15]$ hint the possible role of genetic pleiotropy, a phenomenon in which a genetic variant(s) influence two or more traits [16-18]. Moreover, single nucleotide polymorphisms (SNPs) associated with adulthood BMI exert their influence on adiposity during childhood [15, 19-21]. Twelve out of 15 SNPs associated with childhood BMI at a genome-wide level of significance are also associated with BMI in adults [15]. 
Further interrogation of the National Human Genome Research Institute-EBI (NHGRI-EBI) genome-wide association study (GWAS) catalog [22] reveals that some childhood BMI loci are associated with other measures of adulthood adiposity such as hip circumference, waist circumference, body fat distribution, and fat body mass, energy intake, and type 2 diabetes (ADCY3, ZNF646P1, MC4R, GPR61, BRINP2, FTO) [23-27]. These observations highlight the roles of shared genetic effects; however, to date, the extent of genetic pleiotropy between childhood BMI and a wide range of adult cardiometabolic diseases has not been investigated.

In the present study, we performed a comprehensive analysis of genome-wide summary statistics data for childhood BMI and 15 adult cardiometabolic disease traits (hereafter referred to as adult traits) with the following aims: (1) to test for genetic pleiotropy and enrichment of functional loci in childhood BMI and adult trait pairs, (2) to identify genetic variants associated with childhood BMI and an adult trait, and (3) to investigate the regulatory functions of the identified loci and gain additional insights into the underlying common mechanisms and molecular pathways linking childhood BMI and adult traits.

\section{Results}

\section{Effect of genetic pleiotropy in childhood BMI and} adult traits

Our analyses involved childhood BMI and 15 adult traits (BMI, waist-to-hip ratio, waist circumference, type 2 diabetes, fasting plasma glucose, fasting plasma insulin, glycated hemoglobin, insulin secretion, insulin sensitivity, coronary artery disease, myocardial infarction, low-density lipoprotein (LDL) cholesterol, high-density lipoprotein (HDL) cholesterol, total cholesterol, and triglycerides) (Additional file 1: File S1). We observed evidence for genetic pleiotropy between childhood BMI and adult traits for 13 out of 15 adult traits (except fasting plasma glucose and insulin secretion) (empirical $P<3.33 \times 10^{-3}$; binomial test $P=0.004$ ) (Table 1 , Additional file 1: File S2). Variants associated with higher childhood BMI were associated with increased adult BMI, waist circumference, waist-to-hip ratio, triglycerides, type 2 diabetes risk, myocardial infarction risk, and lower HDL.

\section{Enrichment of functional annotations}

In 14 out of 15 childhood BMI-adult trait tests, SNPs associated with both childhood BMI and adult traits were more likely to be functionally deleterious than SNPs associated with neither traits $\left(q_{11} / q_{00}\right.$ ranging from 1.004 to $7.31 ; P<3.33 \times 10^{-3}$ ) (Table 1, Additional file 1 : File S2). Notably, enrichment of functional deleteriousness was stronger for SNPs associated with both childhood BMI and adult traits than SNPs associated only with childhood BMI or only with adult traits in four childhood BMI-adult trait pairs (coronary artery disease, hemoglobin $\mathrm{A} 1 \mathrm{C}$, insulin secretion, and myocardial infarction). The enrichment folds (s.e.) for SNPs associated with childhood BMI-adult trait vs. SNPs associated with adult trait only vs. SNPs associated with childhood BMI only were as follows: $1.60(0.13)$ vs. $1.38(0.12)$ vs. 1.29 (0.06) for coronary artery disease; $2.52(0.22)$ vs. 0.91 $(0.23)$ vs. 1.22 (0.07) for hemoglobin A1C; 7.31 (1.79) vs. $1.11(0.39)$ vs. $1.33(0.05)$ for insulin secretion; and 1.68 (0.14) vs. $1.11(0.19)$ vs. 1.27 (0.06) for myocardial infarction (Fig. 1, Additional file 1: File S2).

\section{Genetic loci with pleiotropic effects}

The frequency distribution of SNPs associated with childhood BMI only, with adult traits only, and with both childhood BMI and adult traits is shown in Fig. 2 and Additional file 1: File S3. Out of all SNPs that were significantly associated with either or both childhood BMI and an adult trait, the proportions of SNPs commonly associated with both traits were $61.54 \%$ for waist circumference, $47.25 \%$ for BMI, $26.32 \%$ for waist-to-hip ratio, $10.47 \%$ for type 2 diabetes, $4.68 \%$ for coronary artery disease, $4.67 \%$ for HDL cholesterol, $2.87 \%$ for myocardial infarction, $1.68 \%$ for triglycerides, and $0.07 \%$ for total cholesterol. Of the total number of SNPs associated with childhood BMI, 97.07\% were shared with adult BMI. Of the total number of SNPs associated with adult BMI, 47.93\% overlapped with childhood BMI (Fig. 2, Additional file 1: File S3).

A total of 40 loci were associated with childhood BMI and at least one of the following 9 adult traits: BMI, coronary artery disease, HDL cholesterol, myocardial infarction, type 2 diabetes, total cholesterol, triglycerides, waist circumference, and waist-to-hip ratio (Additional file 1: File S4). Of the 40 loci, 39 loci map to previously known GWAS signals associated with childhood BMI and the adult traits tested $\left(P<5 \times 10^{-8}\right.$ in the NHGRI-EBI GWAS catalog: www.ebi.ac.uk/gwas/). One locus (rs12446632 G, near GPRC5B-GPR139) significantly associated with higher childhood BMI and lower adult HDL cholesterol in our study (Fig. 3) is a known GWAS locus for childhood BMI [15] but has been only suggestively associated with HDL cholesterol in previous GWAS [28]. In further functional follow-up analysis, we observed that rs12446632 was cis-eQTL with expression of the KNOP1, GPRC5B, and IQCK genes in a wide range of tissues (Additional file 1 : File S5). The SNP has relatively high functional deleteriousness $(\mathrm{CADD}=10.96)$, and it is within promotor histone marks in gastrointestinal tract mucosa and HepG2 hepatocellular carcinoma cell lines (Haploreg). 
Table 1 Genetic pleiotropy and enrichment of functional deleteriousness among genetic loci associated with childhood BMI and adult cardiometabolic traits

\begin{tabular}{|c|c|c|c|c|c|c|}
\hline \multirow{2}{*}{$\begin{array}{l}\text { Adult cardiometabolic } \\
\text { trait }\end{array}$} & \multicolumn{3}{|c|}{ Genetic pleiotropy } & \multicolumn{3}{|c|}{ Functional annotation enrichment } \\
\hline & $\pi_{11}$ (s.e.) & Pleiotropy test statistics & $P$ value & $\pi_{11}$ (s.e.) & Annotation test statistics & $P$ value \\
\hline Body mass index & $0.055(0.001)$ & $23,690.45$ & $<10^{-300}$ & $1.30(0.04)$ & 173.01 & $2.85 \times 10^{-37}$ \\
\hline Coronary artery disease & $0.01(0)$ & 1215.77 & $2.29 \times 10^{-266}$ & $1.60(0.13)$ & 87.27 & $8.47 \times 10^{-19}$ \\
\hline Fasting glucose & $0(0.001)$ & 1.21 & 0.27 & $0.04(2.74)$ & 6.44 & 0.09 \\
\hline Fasting insulin & $0.047(0.003)$ & 509.74 & $7.24 \times 10^{-113}$ & $1.004(0.22)$ & 41.46 & $5.23 \times 10^{-09}$ \\
\hline Hemoglobin A1c & $0.006(0)$ & 87.12 & $1.02 \times 10^{-20}$ & $2.52(0.22)$ & 48.73 & $1.49 \times 10^{-10}$ \\
\hline HDL cholesterol & $0.005(0)$ & 2151.79 & $<10^{-300}$ & $1.54(0.12)$ & 88.39 & $4.86 \times 10^{-19}$ \\
\hline Insulin secretion & $0(0)$ & 0.89 & 0.35 & $7.31(1.79)$ & 38.69 & $2.01 \times 10^{-08}$ \\
\hline Insulin sensitivity & $0.013(0.001)$ & 393.57 & $1.38 \times 10^{-87}$ & $1.54(0.21)$ & 45.63 & $6.81 \times 10^{-10}$ \\
\hline LDL cholesterol & $0.003(0)$ & 739.01 & $9.85 \times 10^{-163}$ & $1.29(0.20)$ & 98.53 & $3.22 \times 10^{-21}$ \\
\hline Myocardial infarction & $0.01(0)$ & 887.48 & $5.16 \times 10^{-195}$ & $1.68(0.14)$ & 58.51 & $1.22 \times 10^{-12}$ \\
\hline Type 2 diabetes & $0.007(0)$ & 855.26 & $5.23 \times 10^{-188}$ & $1.37(0.19)$ & 65.95 & $3.14 \times 10^{-14}$ \\
\hline Total cholesterol & $0.005(0)$ & 1186.54 & $5.14 \times 10^{-260}$ & $1.45(0.15)$ & 124.99 & $6.47 \times 10^{-27}$ \\
\hline Triglycerides & $0.003(0)$ & 1005.09 & $1.40 \times 10^{-220}$ & $1.44(0.17)$ & 74.82 & $3.97 \times 10^{-16}$ \\
\hline Waist circumference & $0.026(0)$ & $14,809.27$ & $<10^{-300}$ & $1.43(0.06)$ & 112.69 & $2.89 \times 10^{-24}$ \\
\hline Waist-to-hip ratio & $0.019(0)$ & 5271.53 & $<10^{-300}$ & $1.60(0.08)$ & 113.88 & $1.61 \times 10^{-24}$ \\
\hline
\end{tabular}

$\pi_{11}$ is the probability of association of SNPs with both tested traits. $q_{11} / q_{00}$ is the ratio of the probability of jointly associated SNPs being functionally annotated to the probability of a null SNP (associated with neither trait) being functionally annotated

Enrichment for biological pathways and drug ontologies

The set of genes associated with childhood BMI and adult traits were significantly enriched for several biological pathways. The top five most significantly enriched canonical pathways included IL-1 signaling (ratio $=3.26 \%, \quad P=1.47 \times 10^{-6}$ ), androgen signaling (ratio $\left.=2.7 \%, \quad P=2.81 \times 10^{-6}\right)$, corticotropin-releasing hormone signaling $\quad\left(\right.$ ratio $\left.=2.65 \%, \quad P=2.97 \times 10^{-6}\right)$, thrombin signaling (ratio $=1.96 \%, P=6.93 \times 10^{-7}$ ), and molecular mechanisms of cancer (ratio $=1.27 \%, P=$ $6.14 \times 10^{-7}$ ) (Additional file 1: File S6). Ontological analysis found enrichment for disease annotations related to body weight $\left(P=10^{-11} ; \mathrm{FDR}=1.5 \times 10^{-8}\right)$, obesity $(P=$ $\left.1.61 \times 10^{-7} ; \mathrm{FDR}=1.21 \times 10^{-4}\right)$, and schizophrenia $(P=$

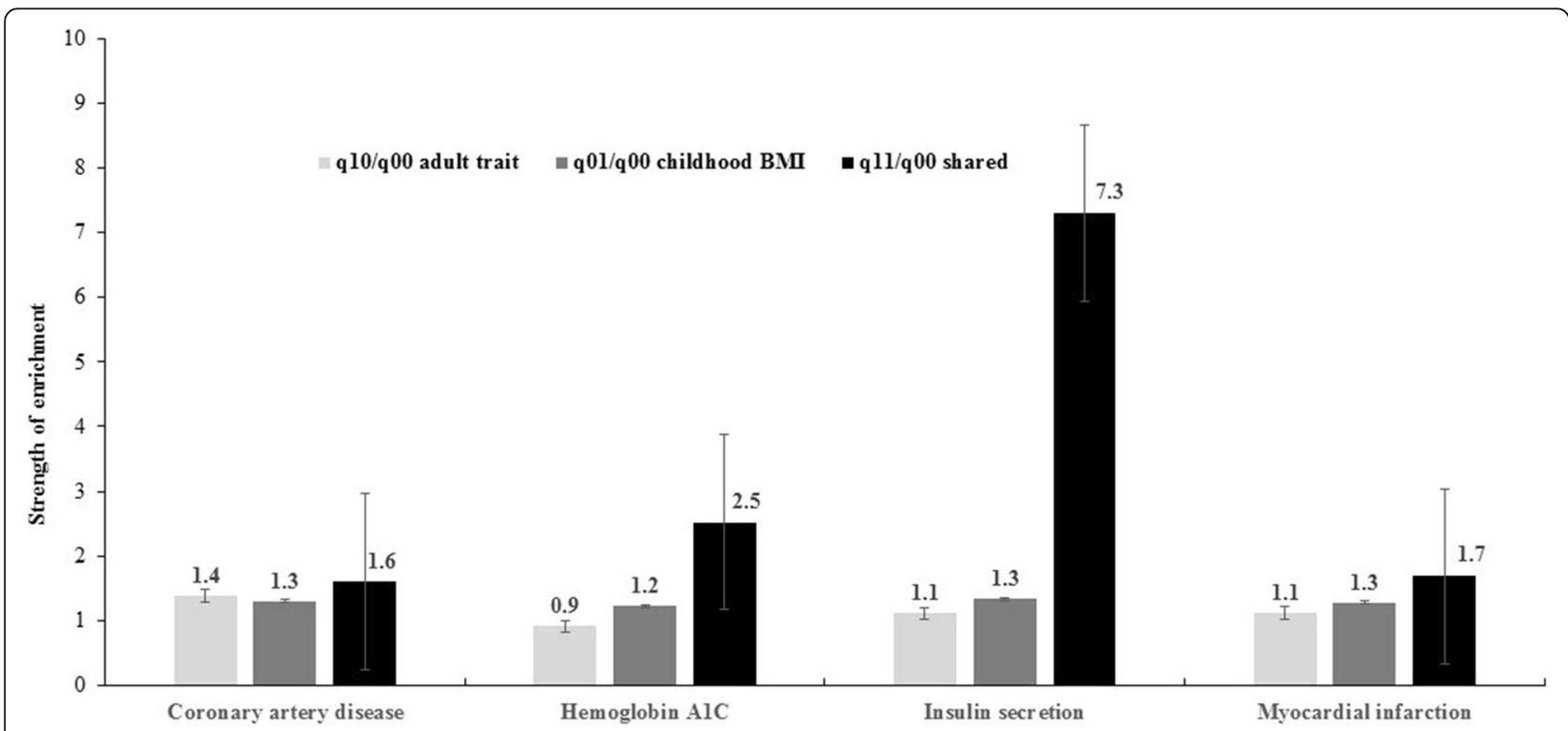

Fig. 1 Enrichment of functional annotations for variants associated with childhood BMI and adult cardiometabolic traits. Vertical lines crossing the bars represent standard error. $q_{01} / q_{00}, q_{10} / q_{00}$, and $q_{11} / q_{00}$ represent the ratio of the probability of SNPs associated with adult traits, child traits, and both traits, respectively, being functionally annotated to the probability of a null SNP being functionally annotated 


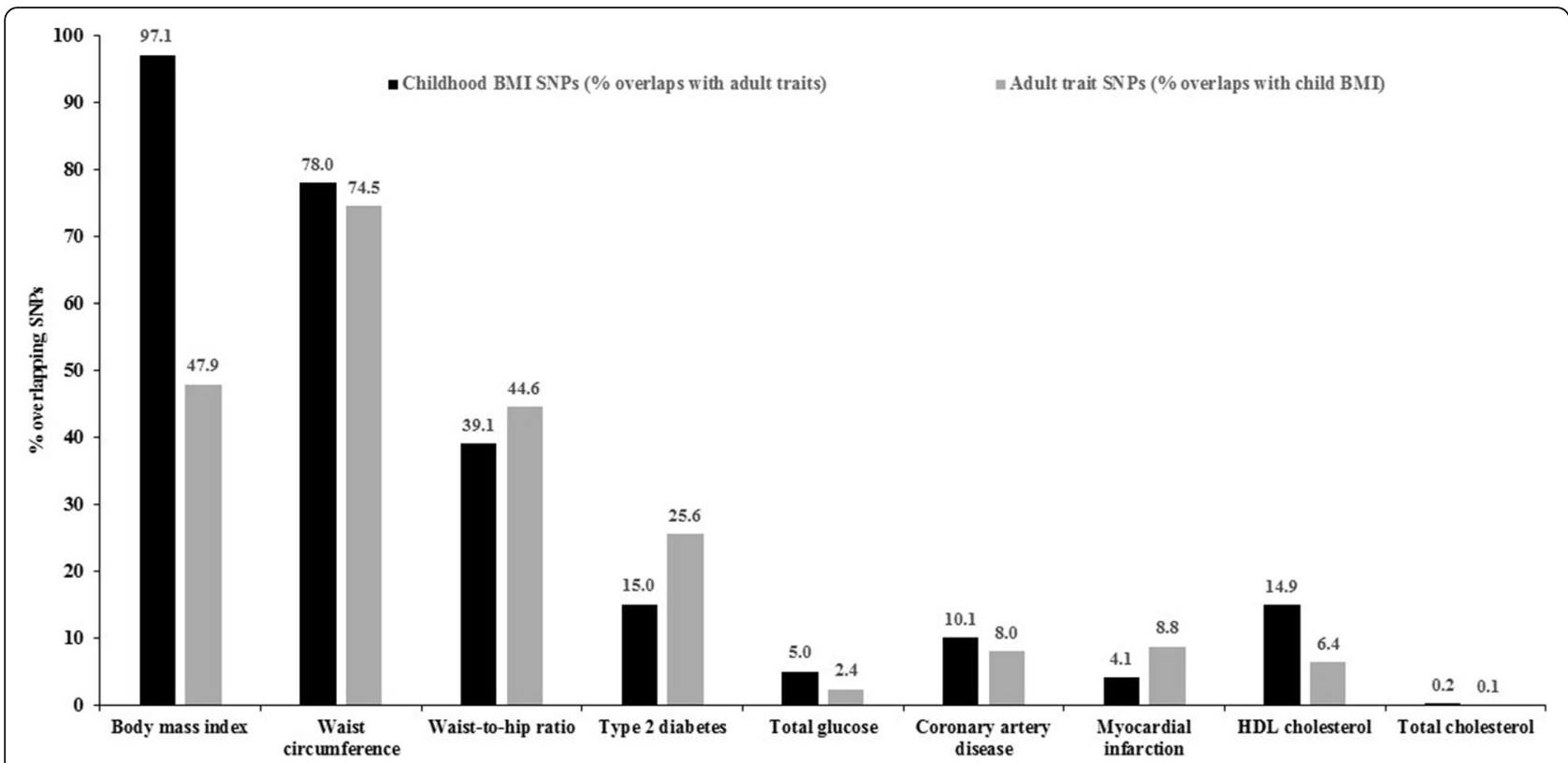

Fig. 2 Percentage of SNPs associated with both childhood BMI and adult cardiometabolic traits out of all SNPs associated with both traits

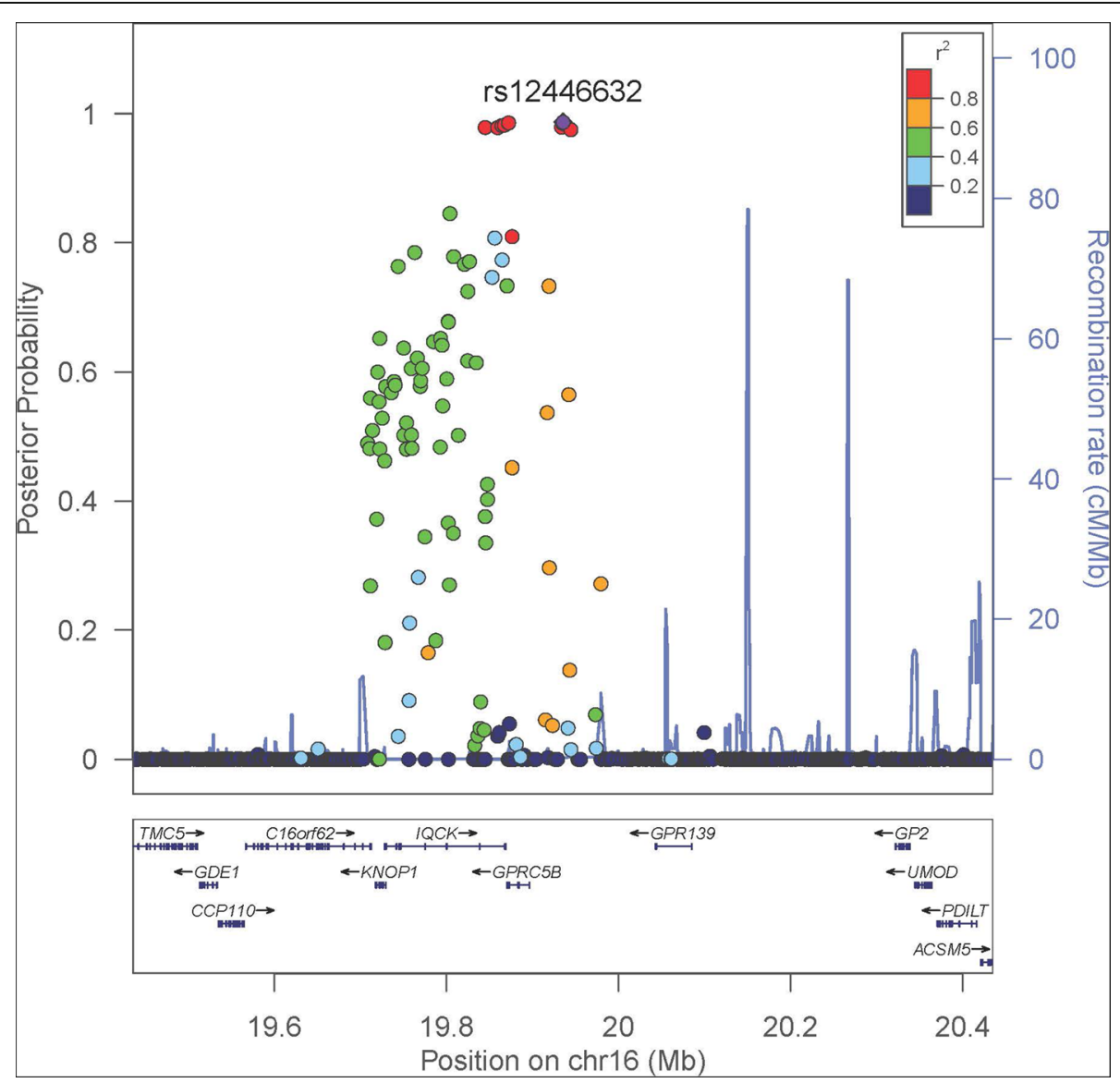

Fig. 3 Regional association plot of the GPRC5B-GPR139 locus significantly associated with childhood BMl and adult HDL cholesterol. Data span $500 \mathrm{~kb}$ centered at the index SNP rs12446632. The $x$-axis denotes genomic position and the $y$-axis denotes the posterior probability of association and recombination rate (cM/Mb). The purple circle point represents the index SNP. The color of each data point indicates its linkage disequilibrium value $\left(r^{2}\right)$ with the index SNP based on HapMap2 
$5.45 \times 10^{-5}$; FDR $=2.73 \times 10^{-2}$ ), as well as enrichment for annotations of the drug ontologies related to low energy diets $\left(P=2.26 \times 10^{-5} ; \mathrm{FDR}=2.93 \times 10^{-2}\right)$ (Additional file 1 : File $\mathrm{S} 7)$.

\section{Discussion}

The present study comprehensively assessed genetic pleiotropic effects in childhood BMI and adult cardiometabolic diseases and showed evidence for shared genetic influence in childhood adiposity and adult chronic diseases. The study also found that SNPs with known biological functions are more likely to be associated with both childhood BMI and adult traits compared to SNPs that are not functional. We replicated 39 genetic loci that are known to be associated with childhood BMI and adult traits in previous GWAS. Additionally, we found novel association of rs12446632 in the GPRC5B-GPR139 locus with adult HDL cholesterol. In all, the findings of the study provide evidence for common genetic mechanisms underlying childhood adiposity and development of cardiometabolic outcomes, thereby facilitating discovery of therapeutic and preventive targets to improve cardiometabolic health across the life span.

Although no genome-wide evaluation of genetic pleiotropy in childhood BMI and a range of adult traits has been done, cross-trait evaluation of individual GWAS loci and genetic risk scores derived from the loci presented in this study have been evaluated in relation to childhood and adulthood BMI. These studies have found that adult BMI loci also operate during childhood $[20,29-33]$. Out of the 97 known adult BMI SNPs discovered in a more recent large-scale GWAS, 86 SNPs had directionally similar effect on childhood BMI and 50 were nominally associated with childhood BMI [15]. The two most recent childhood BMI GWAS studies have reported that seven out of eight loci [34], and 12 out of 15 loci associated with childhood BMI [15], are also associated with BMI in adults. Meanwhile, a strong genetic correlation between childhood BMI and adult BMI has been observed $(\rho=0.73)$ [15]. A few other studies have also reported genetic loci that overlap in their associations with childhood BMI or obesity, adult type 2 diabetes $(F T O, I D-H H E X)[26,35]$, bone mineral density (SP7) [36], waist circumference (TNKS-MSRA) [37], and triglyceride levels (TNKS-MSRA) [38].

Our study found several SNPs that overlapped in their associations with childhood BMI and adult adiposity traits, type 2 diabetes, coronary artery disease, and HDL cholesterol. Notably, the overwhelming majority of childhood BMI genetic loci continue to exert influence on adult BMI. Therefore, genetic factors may partly explain the widely known observation that childhood BMI tracks through adulthood [39]. Our finding also strengthens previous observations of substantial overlaps in the genetic architecture of childhood and adulthood obesity [20, 29-33]. Furthermore, we found that the genetic architecture of childhood BMI is mirrored to a large extent by adult waist circumference (61.54\% overlapping SNPs associated with childhood BMI) and BMI (47.25\% overlapping SNPs) but to a lesser extent by waist-to-hip ratio (26.32\% overlapping SNPs). Similarly, previous studies have shown that a higher adult BMI genetic risk score $[19,20]$, but not a higher waist-to-hip ratio genetic risks score [20], is associated with higher childhood BMI. Future studies interrogating the influence of adult waist circumference genetic loci on childhood adiposity will have the potential to provide new insights into mechanistic underpinnings of the early origins of total body and visceral adiposity.

The association of rs12446632 (near GPRC5B) with childhood BMI-adult HDL cholesterol in our study is noteworthy. A previous GWAS has already reported its association with childhood BMI [15]. The novelty of our finding is the association of rs12446632 with adult HDL cholesterol; the SNP or its proxies (in strong linkage-disequilibrium) fell short of genome-wide significance in previous GWAS of HDL cholesterol [28]. The allele associated with increased childhood BMI was also associated with lower HDL cholesterol, consistent with observational studies that found inverse correlations between childhood BMI and adult HDL cholesterol levels [4, 40]. SNP rs12446632 may have important functional roles given its proximity $(39 \mathrm{kbp})$ to the $5^{\prime}$ of $G P R C 5 B$, its relatively high CADD deleteriousness score, and evidence of roles in regulating expression of $G P R C 5 B$, and overlapping histone marks. GPRC5B is highly expressed in adipose tissue and the central nervous system [41]. The encoded protein is a lipid raft-associated transmembrane protein that may be critical for inflammatory signaling in adipose tissue [41, 42] and modulates insulin secretion [43]. We also observed that rs12446632 was associated with adulthood adiposity measures consistent with previous GWAS that reported significant associations of the SNP with adulthood BMI [21, 44, 45], obesity [46], waist circumference [23], and hip circumference [23]. Given the known association of adulthood BMI with dyslipidemia [47] and this widely replicated association of the SNP with obesity [21, 23, 44-46], it will be noteworthy to investigate whether the rs12446632-adult HDL cholesterol association found in our study is mediated through BMI during adulthood.

Our findings demonstrating significant enrichment of pathways such as IL-1 signaling, androgen signaling, corticotropin-releasing hormone signaling, and thrombin signaling highlight the possibility of the 
relationships between childhood adiposity and adulthood cardiometabolic diseases. These relationships may involve mechanisms broader than endothelial dysfunction, insulin resistance, inflammation, and adipocytokines [48, 49]. More detailed understanding of the pathways in which childhood adiposity and adult cardiometabolic disease traits overlap could provide new avenues for therapeutic targeting. This seems promising given our observation of enrichment for metabolic disease-related ontologies and potential drug targets among the set of genes jointly associated with childhood BMI and adult traits. Furthermore, our study showed that genetic variants associated with increased childhood adiposity tend to increase the risk of obesity, cardiovascular diseases, type 2 diabetes, and dyslipidemia in adulthood. This finding suggests that prevention of childhood obesity informed by genetic evidence will be beneficial to lower cardiometabolic disease risk in later life.

We acknowledge that there are limitations to our study. Despite the large sample sizes of the consortia-based meta-analysis studies, there were differences in sample size and number of SNPs among the different studies. As a result, the traits for which the source studies had relatively fewer loci and samples were likely to be less enriched for SNPs with potential shared influence (e.g., fasting glucose). In addition, some of the observed associations may not be due to independent effects of the same locus on childhood BMI and an adult trait, but due to the correlation of traits that are in the causal pathway or through other unmeasured traits. An important strength of our study is the integrated modeling of functional annotation and GWAS summary statistics data from pairs of traits. This multi-trait approach has been instrumental in testing for functional enrichment and detection of novel loci with multi-trait effects [50]. Implementation of this approach considerably expanded our understanding of the genetic links between childhood BMI and adult traits.

\section{Conclusions}

The present study found pleiotropic genetic effects on childhood obesity and adult cardiometabolic diseases. The previously identified genetic loci, including our novel loci with pleiotropic effects, were functionally enriched for biological pathways related to adiposity and cardiovascular diseases. These biological pathways through which the genes operate provide the potential to disentangle the genetic basis of life course associations between childhood obesity and adult cardiometabolic diseases.

\section{Methods}

\section{Data sets}

We assembled GWAS summary statistics data including $P$ values and effect directions of genome-wide SNPs reported by six consortia [21, 23, 28, 51-57], involving childhood BMI and 15 adult traits (BMI, waist-to-hip ratio, waist circumference, type 2 diabetes, fasting plasma glucose, fasting plasma insulin, glycated hemoglobin, insulin secretion, insulin sensitivity, coronary artery disease, myocardial infarction, LDL cholesterol, HDL cholesterol, total cholesterol, and triglycerides). The majority of the study participants had European ancestry, with some studies additionally involving individuals of East Asian, South Asian, and Hispanic- and African-Americans (Additional file 1: File S1).

\section{Functional annotation of SNPs}

Functional annotation of SNPs was carried out using the Combined Annotation Dependent Depletion (CADD) framework as implemented in CADD v1.2 (http://cadd. gs.washington.edu) [58]. CADD integrates functional and evolutionary importance from multiple annotation sources into one metric by contrasting variants that survived natural selection with simulated mutations to generate a deleteriousness score for each genetic variant. Variants with Phred-like CADD score $(-10 * \log 10$ [rank/ total]) values $\geq 15$ were considered deleterious [58] and were assigned annotation of 1 , and those with CADD score values $<15$ were assigned annotation of 0 . The assigned annotation values were used as inputs in annotation tests. Subsequent annotation tests assessed functional enrichment among (1) SNPs associated only with childhood BMI compared to SNPs associated with neither trait (estimated by $q_{10} / q_{00}$ ), (2) SNPs associated only with an adult trait compared to SNPs associated with neither trait $\left(q_{01} / q_{00}\right)$, and (3) SNPs associated with both childhood BMI and an adult trait compared to SNPs associated with neither trait $\left(q_{11} / q_{00}\right) \cdot q_{11} / q_{00}$ is the ratio of the probability of jointly associated SNPs being functionally annotated to the probability of a null SNP (associated with neither trait) being functionally annotated [50].

\section{Tests for genetic pleiotropy}

We tested for evidence of pleiotropy, enrichment of functional annotation, and association of SNPs with both childhood BMI and an adult trait using the GPA v1.1-0 $\mathrm{R}$ package [50]. GPA (Genetic analysis incorporating Pleiotropy and Annotation) implements a unified statistical approach that integrates pleiotropy and functional annotation data and tests for enrichment of annotations from functional datasets in variants associated with pairs of traits. A total of 15 childhood BMI-adult trait pair tests were performed. 
All tests were conducted under the false discovery rate control (FDR) at the 0.05 level using 10,000 expectation maximization iterations. Evidence for enrichment of pleiotropy and functional annotation were considered significant at the Bonferroni-corrected level $P$ value $=$ $3.33 \times 10^{-3}(0.05 / 15$ tests). SNPs were considered to be significantly associated with both traits in a childhood BMI-adult trait pair if posterior probability of association was $>0.95$ with FDR of 0.05 as implemented in GPA [50]. When two or more SNPs within a $1 \mathrm{Mb}$ region were associated with a trait pair, the index SNP with the highest posterior probability of association and other SNPs not in LD with the index SNP $\left(r^{2}<0.06\right.$ in the 1000 Genomes Phase $3 \mathrm{CEU}$ population sample) were considered to be independent associations. SNPs that were newly identified to be associated with childhood $\mathrm{BMI}$ and/or adult traits were examined for potential regulatory effect on gene expression level in different tissues using the Genotype-Tissue Expression (GTEx v. 6) [59] database. Possible regulatory effects of the lead SNPs were assessed by examining if the SNPs are within promoters, enhancers, DNAse, and transcription factor binding using the Haploreg tool (version 4.1) [60].

\section{Ontology analysis and drug target annotations}

We performed ontology analysis on the list of genes mapping to loci associated with childhood BMI-adult trait for connectivity using the online tool WEB-based GEne SeT AnaLysis Toolkit (WebGestalt) [61]. The hypergeometric distribution was used to test for statistical significance. Adjustment for multiple testing was controlled using the Benjamini-Hochberg procedure.

\section{Pathway analysis}

To determine whether the list of genes showing significant association with childhood BMI-adult traits was enriched in biological functions or processes relevant to those traits, we looked for enrichment of biological pathways using the Ingenuity Pathway Analysis (IPA) bioinformatics resource (IPA, Qiagen, Redwood City, CA, USA). We examined the overlap of the pleiotropic gene list with gene sets representing canonical pathways in IPA for the genes associated with childhood BMI and adult traits. Fisher's exact test was used to assess the statistical significance of the overlap between our list of pleiotropic genes submitted to IPA and the list of genes in canonical pathways in the databases.

\section{Additional file}

Additional file 1: File S1. GWAS summary statistics profile of traits and diseases analyzed in the study. File S2. Genetic pleiotropic effects and enrichment of functionally deleterious SNPs associated with childhood BMI-adult cardiometabolic traits. File S3. Number of SNPs associated only with childhood BMI, only with an adult cardiometabolic trait, and both childhood BMI and an adult cardiometabolic trait with posterior probability $>0.95$. File S4. Genetic loci significantly associated with both childhood BMI and adult cardiometabolic traits with posterior probability $>0.95$. File S5. Genes whose expression levels were significantly associated with SNP rs12446632. File S6. Canonical pathways significantly enriched in genes associated with childhood BMl-adult cardiometabolic traits. File S7. Significantly over-represented disease and drug ontologies in the set of genes associated with childhood BMl-adult cardiometabolic traits. (DOCX $282 \mathrm{~kb}$ )

\section{Abbreviations}

BMI: Body mass index; CADD: Combined Annotation Dependent Depletion; eQTL: Expression quantitative trait locus; GPA: Genetic analysis incorporating Pleiotropy and Annotation; GTEx: Genotype-Tissue Expression; GWAS: Genome-wide association study; HDL: High-density lipoprotein; IPA: Ingenuity Pathway Analysis; LD: Linkage disequilibrium; LDL: Low-density lipoprotein; SNP: Single nucleotide polymorphism; WebGestalt: WEB-based GEne SeT AnaLysis Toolkit

\section{Acknowledgements}

Not applicable.

\section{Funding}

This research was supported by the Intramural Research Program of the Eunice Kennedy Shriver National Institute of Child Health and Human Development, National Institutes of Health. Additional support was obtained from the National Institute on Minority Health and Health Disparities. This work utilized the computational resources of the NIH HPC Biowulf cluster (http://hpc.nih.gov).

\section{Availability of data and materials}

The data analyzed in this study are available online. Additional file 1: File S1 lists the URL of the data sources.

\section{Authors' contributions}

FT-A conceived and designed this study. AL gathered and managed the data. AL and FT-A analyzed the data. FT-A wrote the paper. AL, TW, and KSP provided critical intellectual content. All authors approved the final manuscript.

Ethics approval and consent to participate

The samples met the consent criteria established by the projects that participated in the six consortia that assembled the genotypes [21, 23, 28, 51-57]. The NIH Office of Human Subjects Research Program granted the study an exemption from IRB review (OHSRP ID Number: 18-NICHD-00412) per 45 CFR 46 and $\mathrm{NIH}$ policy for the use of specimens/data.

\section{Consent for publication}

Not applicable.

\section{Competing interests}

The authors declare that they have no competing interests.

\section{Publisher's Note}

Springer Nature remains neutral with regard to jurisdictional claims in published maps and institutional affiliations.

\section{Author details}

'Epidemiology Branch, Division of Intramural Population Health Research, Eunice Kennedy Shriver National Institute of Child Health and Human Development, National Institutes of Health, 6710B Rockledge Drive, Room 3204, Bethesda, MD 20892-7004, USA. ${ }^{2}$ Laboratorio de Endocrinologia Molecular, Hospital Juárez de México, Mexico City, Mexico. 


\section{Received: 8 November 2018 Accepted: 20 March 2019} Published online: 04 April 2019

\section{References}

1. Ng M, Fleming T, Robinson M, Thomson B, Graetz N, Margono C, et al. Global, regional, and national prevalence of overweight and obesity in children and adults during 1980-2013: a systematic analysis for the Global Burden of Disease Study 2013. Lancet. 2014;384(9945):766-81.

2. Zhang T, Zhang H, Li Y, Sun D, Li S, Fernandez C, et al. Temporal relationship between childhood body mass index and insulin and its impact on adult hypertension: the Bogalusa Heart Study. Hypertension. 2016;68(3):818-23.

3. Tirosh A, Shai I, Afek A, Dubnov-Raz G, Ayalon N, Gordon B, et al. Adolescent BMI trajectory and risk of diabetes versus coronary disease. N Engl J Med. 2011;364(14):1315-25.

4. Sinaiko AR, Donahue RP, Jacobs DR Jr, Prineas RJ. Relation of weight and rate of increase in weight during childhood and adolescence to body size, blood pressure, fasting insulin, and lipids in young adults. The Minneapolis Children's Blood Pressure Study. Circulation. 1999;99(11):1471-6.

5. Simmonds M, Llewellyn A, Owen CG, Woolacott N. Predicting adult obesity from childhood obesity: a systematic review and meta-analysis. Obes Rev. 2016;17(2):95-107.

6. Simmonds M, Burch J, Llewellyn A, Griffiths C, Yang H, Owen C, et al. The use of measures of obesity in childhood for predicting obesity and the development of obesity-related diseases in adulthood: a systematic review and meta-analysis. Health Technol Assess. 2015:19(43):1-336.

7. Raitakari OT, Juonala M, Kahonen M, Taittonen L, Laitinen T, Maki-Torkko N et al. Cardiovascular risk factors in childhood and carotid artery intimamedia thickness in adulthood: the Cardiovascular Risk in Young Finns Study. JAMA. 2003;290(17):2277-83.

8. Morrison JA, Friedman LA, Wang P, Glueck CJ. Metabolic syndrome in childhood predicts adult metabolic syndrome and type 2 diabetes mellitus 25 to 30 years later. J Pediatr. 2008;152(2):201-6.

9. Lai CC, Sun D, Cen R, Wang J, Li S, Fernandez-Alonso C, et al. Impact of long-term burden of excessive adiposity and elevated blood pressure from childhood on adulthood left ventricular remodeling patterns: the Bogalusa Heart Study. J Am Coll Cardiol. 2014;64(15):1580-7.

10. Juonala M, Magnussen CG, Berenson GS, Venn A, Burns TL, Sabin MA, et al. Childhood adiposity, adult adiposity, and cardiovascular risk factors. N Engl Med. 2011;365(20):1876-85

11. Freedman DS, Patel DA, Srinivasan SR, Chen W, Tang R, Bond MG, et al. The contribution of childhood obesity to adult carotid intima-media thickness: the Bogalusa Heart Study. Int J Obes. 2008;32(5):749-56.

12. Davis PH, Dawson JD, Riley WA, Lauer RM. Carotid intimal-medial thickness is related to cardiovascular risk factors measured from childhood through middle age: The Muscatine Study. Circulation. 2001;104(23):2815-9.

13. Baker JL, Olsen LW, Sorensen TI. Childhood body-mass index and the risk of coronary heart disease in adulthood. N Engl J Med. 2007;357(23):2329-37.

14. Bulik-Sullivan B, Finucane HK, Anttila V, Gusev A, Day FR, Loh PR, et al. An atlas of genetic correlations across human diseases and traits. Nat Genet. 2015;47(11):1236-41.

15. Felix JF, Bradfield JP, Monnereau C, van der Valk RJ, Stergiakouli E, Chesi A, et al. Genome-wide association analysis identifies three new susceptibility loci for childhood body mass index. Hum Mol Genet. 2016;25(2):389-403.

16. Stearns FW. One hundred years of pleiotropy: a retrospective. Genetics. 2010;186(3):767-73.

17. Sivakumaran S, Agakov F, Theodoratou E, Prendergast JG, Zgaga L, Manolio $\mathrm{T}$, et al. Abundant pleiotropy in human complex diseases and traits. Am J Hum Genet. 2011;89(5):607-18.

18. Pickrell JK, Berisa T, Liu JZ, Segurel L, Tung JY, Hinds DA. Detection and interpretation of shared genetic influences on 42 human traits. Nat Genet. 2016:48(7):709-17.

19. Monnereau C, Vogelezang S, Kruithof CJ, Jaddoe WW, Felix JF. Associations of genetic risk scores based on adult adiposity pathways with childhood growth and adiposity measures. BMC Genet. 2016;17(1):120.

20. Vogelezang S, Monnereau C, Gaillard R, Renders CM, Hofman A, Jaddoe WW, et al. Adult adiposity susceptibility loci, early growth and general and abdominal fatness in childhood: the Generation R Study. Int J Obes. 2015;39(6):1001-9.
21. Locke AE, Kahali B, Berndt SI, Justice AE, Pers TH, Day FR, et al. Genetic studies of body mass index yield new insights for obesity biology. Nature. 2015;518(7538):197-206.

22. MacArthur J, Bowler E, Cerezo M, Gil L, Hall P, Hastings E, et al. The new NHGRI-EBI catalog of published genome-wide association studies (GWAS catalog). Nucleic Acids Res. 2017:45(D1):D896-901.

23. Shungin D, Winkler TW, Croteau-Chonka DC, Ferreira T, Locke AE, Magi R, et al. New genetic loci link adipose and insulin biology to body fat distribution. Nature. 2015;518(7538):187-96.

24. Lu Y, Day FR, Gustafsson S, Buchkovich ML, Na J, Bataille V, et al. New loci for body fat percentage reveal link between adiposity and cardiometabolic disease risk. Nat Commun. 2016;7:10495.

25. Pei YF, Zhang L, Liu Y, Li J, Shen H, Liu YZ, et al. Meta-analysis of genomewide association data identifies novel susceptibility loci for obesity. Hum Mol Genet. 2014:23(3):820-30.

26. Imamura M, Takahashi A, Yamauchi T, Hara K, Yasuda K, Grarup N, et al. Genome-wide association studies in the Japanese population identify seven novel loci for type 2 diabetes. Nat Commun. 2016;7:10531.

27. Tanaka T, Ngwa JS, van Rooij FJ, Zillikens MC, Wojczynski MK, Frazier-Wood AC, et al. Genome-wide meta-analysis of observational studies shows common genetic variants associated with macronutrient intake. Am J Clin Nutr. 2013;97(6):1395-402.

28. Willer CJ, Schmidt EM, Sengupta S, Peloso GM, Gustafsson S, Kanoni S, et al. Discovery and refinement of loci associated with lipid levels. Nat Genet. 2013;45(11):1274-83.

29. Zhao J, Bradfield JP, Zhang H, Sleiman PM, Kim CE, Glessner JT, et al. Role of BMI-associated loci identified in GWAS meta-analyses in the context of common childhood obesity in European Americans. Obesity (Silver Spring). 2011;19(12):2436-9.

30. Zhao J, Bradfield JP, Li M, Wang K, Zhang H, Kim CE, et al. The role of obesity-associated loci identified in genome-wide association studies in the determination of pediatric BMI. Obesity (Silver Spring). 2009;17(12):2254-7.

31. den Hoed M, Ekelund U, Brage S, Grontved A, Zhao JH, Sharp SJ, et al. Genetic susceptibility to obesity and related traits in childhood and adolescence: influence of loci identified by genome-wide association studies. Diabetes. 2010:59(11):2980-8.

32. Belsky DW, Moffitt TE, Houts R, Bennett GG, Biddle AK, Blumenthal JA, et al. Polygenic risk, rapid childhood growth, and the development of obesity: evidence from a 4-decade longitudinal study. Arch Pediatr Adolesc Med. 2012;166(6):515-21.

33. Warrington NM, Howe LD, Wu YY, Timpson NJ, Tilling K, Pennell CE, et al. Association of a body mass index genetic risk score with growth throughout childhood and adolescence. PLoS One. 2013;8(11):e79547.

34. Bradfield JP, Taal HR, Timpson NJ, Scherag A, Lecoeur C, Warrington NM, et al. A genome-wide association meta-analysis identifies new childhood obesity loci. Nat Genet. 2012;44(5):526-31.

35. Zhao J, Bradfield JP, Zhang H, Annaiah K, Wang K, Kim CE, et al. Examination of all type 2 diabetes GWAS loci reveals HHEX-IDE as a locus influencing pediatric BMI. Diabetes. 2010;59(3):751-5.

36. Zhao J, Bradfield JP, Li M, Zhang H, Mentch FD, Wang K, et al. BMDassociated variation at the Osterix locus is correlated with childhood obesity in females. Obesity (Silver Spring). 2011;19(6):1311-4.

37. Lindgren CM, Heid IM, Randall JC, Lamina C, Steinthorsdottir V, Qi L, et al. Genome-wide association scan meta-analysis identifies three Loci influencing adiposity and fat distribution. PLoS Genet. 2009;5(6):e1000508.

38. Kathiresan S, Willer CJ, Peloso GM, Demissie S, Musunuru K, Schadt EE, et al. Common variants at 30 loci contribute to polygenic dyslipidemia. Nat Genet. 2009;41(1):56-65.

39. Whitaker RC, Wright JA, Pepe MS, Seidel KD, Dietz WH. Predicting obesity in young adulthood from childhood and parental obesity. N Engl J Med. 1997;337(13):869-73.

40. Freedman DS, Khan LK, Dietz WH, Srinivasan SR, Berenson GS. Relationship of childhood obesity to coronary heart disease risk factors in adulthood: the Bogalusa Heart Study. Pediatrics. 2001;108(3):712-8.

41. Sano T, Kim YJ, Oshima E, Shimizu C, Kiyonari H, Abe T, et al. Comparative characterization of GPRC5B and GPRC5CLacZ knockin mice; behavioral abnormalities in GPRC5B-deficient mice. Biochem Biophys Res Commun 2011:412(3):460-5.

42. Purcell CJ, Stroink G, Horacek BM. Effect of torso boundaries on electric potential and magnetic field of a dipole. IEEE Trans Biomed Eng. 1988;35(9):671-8. 
43. Soni A, Amisten S, Rorsman P, Salehi A. GPRC5B a putative glutamatereceptor candidate is negative modulator of insulin secretion. Biochem Biophys Res Commun. 2013;441(3):643-8.

44. Justice AE, Winkler TW, Feitosa MF, Graff M, Fisher VA, Young K, et al. Genome-wide meta-analysis of 241,258 adults accounting for smoking behaviour identifies novel loci for obesity traits. Nat Commun. 2017;8:14977.

45. Graff M, Scott RA, Justice AE, Young KL, Feitosa MF, Barata L, et al. Genomewide physical activity interactions in adiposity - a meta-analysis of 200,452 adults. PLoS Genet. 2017;13(4):e1006528.

46. Berndt SI, Gustafsson S, Magi R, Ganna A, Wheeler E, Feitosa MF, et al. Genome-wide meta-analysis identifies 11 new loci for anthropometric traits and provides insights into genetic architecture. Nat Genet. 2013;45(5):501-12.

47. Lloyd LJ, Langley-Evans SC, McMullen S. Childhood obesity and risk of the adult metabolic syndrome: a systematic review. Int J Obes. 2012;36(1):1-11.

48. Herouvi D, Karanasios E, Karayianni C, Karavanaki K. Cardiovascular disease in childhood: the role of obesity. Eur J Pediatr. 2013;172(6):721-32.

49. Ayer J, Charakida M, Deanfield JE, Celermajer DS. Lifetime risk: childhood obesity and cardiovascular risk. Eur Heart J. 2015;36(22):1371-6.

50. Chung D, Yang C, Li C, Gelernter J, Zhao H. GPA: a statistical approach to prioritizing GWAS results by integrating pleiotropy and annotation. PLoS Genet. 2014;10(11):e1004787.

51. Horikoshi M, Beaumont RN, Day FR, Warrington NM, Kooijman MN, Fernandez-Tajes J, et al. Genome-wide associations for birth weight and correlations with adult disease. Nature. 2016:538(7624):248-52

52. Replication DIG, Meta-analysis C, Asian Genetic Epidemiology Network Type 2 Diabetes C, South Asian Type 2 Diabetes C, Mexican American Type 2 Diabetes C, Type 2 Diabetes Genetic Exploration by Nex-generation sequencing in muylti-Ethnic Samples C, et al. Genome-wide trans-ancestry meta-analysis provides insight into the genetic architecture of type 2 diabetes susceptibility. Nat Genet. 2014;46(3):234-44.

53. Dupuis J, Langenberg C, Prokopenko I, Saxena R, Soranzo N, Jackson AU, et al. New genetic loci implicated in fasting glucose homeostasis and their impact on type 2 diabetes risk. Nat Genet. 2010;42(2):105-16.

54. Soranzo N, Sanna S, Wheeler E, Gieger C, Radke D, Dupuis J, et al. Common variants at 10 genomic loci influence hemoglobin $\mathrm{A}(1)(\mathrm{C})$ levels via glycemic and nonglycemic pathways. Diabetes. 2010;59(12):3229-39.

55. Prokopenko I, Poon W, Magi R, Prasad BR, Salehi SA, Almgren P, et al. A central role for GRB10 in regulation of islet function in man. PLoS Genet. 2014;10(4):e1004235.

56. Walford GA, Gustafsson S, Rybin D, Stancakova A, Chen H, Liu CT, et al. Genome-wide association study of the modified Stumvoll Insulin Sensitivity Index identifies BCL2 and FAM19A2 as novel insulin sensitivity loci. Diabetes. 2016;65(10):3200-11.

57. Nikpay M, Goel A, Won HH, Hall LM, Willenborg C, Kanoni S, et al. A comprehensive 1,000 genomes-based genome-wide association metaanalysis of coronary artery disease. Nat Genet. 2015;47(10):1121-30.

58. Kircher M, Witten DM, Jain P, O'Roak BJ, Cooper GM, Shendure J. A general framework for estimating the relative pathogenicity of human genetic variants. Nat Genet. 2014;46(3):310-5.

59. Consortium GT. Human genomics. The Genotype-Tissue Expression (GTEx) pilot analysis: multitissue gene regulation in humans. Science. 2015;348(6235):648-60

60. Ward LD, Kellis M. HaploReg: a resource for exploring chromatin states, conservation, and regulatory motif alterations within sets of genetically linked variants. Nucleic Acids Res. 2012;40(Database issue):D930-4.

61. Wang J, Duncan D, Shi Z, Zhang B. WEB-based GEne SeT AnaLysis Toolkit (WebGestalt): update 2013. Nucleic Acids Res. 2013;41(Web Server issue): W77-83.

Ready to submit your research? Choose BMC and benefit from:

- fast, convenient online submission

- thorough peer review by experienced researchers in your field

- rapid publication on acceptance

- support for research data, including large and complex data types

- gold Open Access which fosters wider collaboration and increased citations

- maximum visibility for your research: over $100 \mathrm{M}$ website views per year

At BMC, research is always in progress.

Learn more biomedcentral.com/submissions 